\title{
Universe (Part 2 v2): Rolling of space and time into a point
}

\begin{abstract}
In previously work, 1 this author developed a theory which shows the unknown relations between main parameters in a given field of science. Using this theory, the outcomes of the derived formulas to estimate some magnitudes of our Universe exposed both well-known and new unknown equations. That paper1 which should be considered part 1 of this series offers possibly valid relations between energy, volume, time, matter, distance. The offered research shows that there exists in the Universe ONLY one primary substance - ENERGY. Volume, matter, time, fields are forms of the energy and can be transformed one to other, such as the transformation in the famous formula $E=m c^{2}$.

In paper, part 2 of that series, the author shows that the parameters of space (volume, distance) and time have limits (maximal magnitudes). The time, volume (distance) contract (collapse) into a point under the specific density of the matter, energy, pressure, temperature, frequency, intensity of acceleration, electric, magnetic, fields. The maximal force, temperature is independent from other conditions.

The computed critical values are very importance for micro and macro worlds. When a particle (universe, body) approaches them, for an external observer their dimensions, energy and lifetime tend to zero, i.e. they seem to disappear (they delete into infinity). Because they depend on matter (energy), this means that matter (energy) and in the universe are limited.
\end{abstract}

Volume 2 Issue 6 - 2018

\author{
Alexander A Bolonkin \\ Former Senior Researcher of NASA and Scientific Laboratories \\ of the USA Air Forces, USA

\begin{abstract}
Correspondence: A Bolonkin, Former Senior Researcher of NASA and Scientific Laboratories of the USA Air Forces, 1310 Avenue R, \#6-F, Brooklyn, NY, I I 229, USA, Tel 718-339-4563, Email abolomkin@gmail.com
\end{abstract}

Received: June 13,2018 | Published: November 19, 2018

\section{Introduction}

The Universe is everything that exists, including the stars, the planets, galaxies, matter and energy. The Universe is also fillings time and intergalactic space. The term universe may be used also as the world, the cosmos, or nature.

Astronomers observe current and earlier stages in the development of the Universe. They can see at great distances. They suggest that the universe has always been ruled by the same physical laws. The Solar System is implanted in a galaxy. The most galaxies have the billions of stars. The Milky Way and other galaxies that exist outside it, are located as far as astronomical instruments can reach. Current cosmology studies of galaxies and their spectral lines. The universe is expanding and had a beginning. Telescope Hubble shows galaxies consisting of billions of stars.

In Part $1^{1}$ author offers the relations between volume, time, matter, distance, and energy. The author shows the Universe has only one substance - ENERGY. Time, volume, matter, field's evidence energy and they can be changed one to other. Author gives the equations which allow calculating these transformations. In particularly the author get from his theory the famous formula $E=m c^{2}$.

The author shows that the matter, energy, and fields have boundaries (maximal amount). Volume and time can collapse into points under the specific density of matter, energy, temperature, pressure, frequency, intensity of acceleration, magnetic, electric, fields. The maximal force and temperature are independent from other conditions.

\section{Theory shows: Values of the matter, energy and fields have a boundary. Volume and time collapse in these bounds.}

The author shows the magnitudes of the matter, energy and fields have maximal limits. The volume and time collapse under the specific density of the matter, energy, pressure, temperature, frequency, intensity of acceleration, magnetic, electric, fields.

The well-known numbers used in his expressions are next:

$$
\begin{aligned}
c & =2.997925 \cdot 10^{8} \mathrm{~m} / \mathrm{s}, \quad e=1.60219 \cdot 10^{-19} \quad C, \quad G=6.6743 \cdot 10^{-11} \quad \mathrm{Nm}^{2} / \mathrm{kg}^{2}, \\
\varepsilon_{0} & =\frac{1}{36 \pi \cdot 10^{9}}=8.8542 \cdot 10^{-12} \quad \frac{F}{m}, \quad \mu_{0}=4 \pi \cdot 10^{-7}=1.257 \cdot 10^{-6} \quad \frac{\mathrm{H}}{\mathrm{m}}, \quad \pi=3.141592654 ; \\
h & =6.6261 \cdot 10^{-34} \quad \mathrm{~J} \cdot \mathrm{s}, \quad \hbar=h / 2 \pi, \quad \sigma=5.67032 \cdot 10^{-8} \quad \mathrm{~W} / \mathrm{m}^{2} \mathrm{~K}^{4}, \quad k_{B}=1.38066 \cdot 10^{-23} \quad j / \mathrm{K} .
\end{aligned}
$$

here $e$ - electronic charge, $\mathrm{C} ; c$ - speed of light, $\mathrm{m} / \mathrm{s} ; G$ - gravitation, $\mathrm{Nm}^{2} / \mathrm{kg}^{2} ; \varepsilon_{o}$ - magnetic constant, $\mathrm{H} / \mathrm{m} ; \varepsilon_{o}$ - electric constant, $\mathrm{F} / \mathrm{m}$; $h$ - Planck constant, Js; $\sigma$ - Stefan - Boltzmann constant, $\mathrm{W} / \mathrm{m}^{2} \mathrm{~K}^{4}$.

The author postulated the relations:

$$
\frac{d T}{d T_{0}}=\left(1-\frac{E}{E_{0}}\right)^{1 / 2}=\gamma, \frac{d l}{d l_{0}}=\left(1-\frac{E}{E_{0}}\right)^{1 / 2}=\gamma,
$$

here $T$ is time into given volume having given substances (matter, energy, field, temperature, etc.), sec.; $E_{0}$ is time of outer observer in his outer space, sec; $E$ is energy into the given volume, $\mathrm{J} ; E_{0}$ is maximal possible energy into the given volume (density of energy), $\mathrm{J} / \mathrm{V}\left(V-\right.$ volume, $\left.\mathrm{m}^{3}\right) ; l$ is length in given volume having given substance (matter, energy, field, temperature, etc.) and measured by outer observer, $\mathrm{m} ; l_{0}$ is length into outer space measured by the outer observer (length measured by outer observer), $\mathrm{m}$; is contraction (coagulation, rolling, collapse) coefficient.

The equation (2) for $E_{0}=M c^{2}>0, E / E_{0} \leq 1$ gives the limits of 
values (maximal acceleration, pressure, frequency, temperature, mass and volume density, intensity if fields, event horizons, etc.) which depend from positive mass.

The following expressions (equations for decreasing the length, time from circumstances in the given volume) can be gotten from the connection (2). In this step, we use the equation $E_{0}=M c^{2}$ and the appropriate equations from part 1.

Influence of pressure $\mathrm{N} / \mathrm{m}^{2}$ :

$\gamma=\left(1-\frac{p}{p_{0}}\right)^{1 / 2}=\left(1-\frac{M^{2} G^{3}}{c^{8}} p\right)^{1 / 2}$, where $p_{0}=\frac{c^{8}}{G^{3}} \frac{1}{M^{2}}$,

where $p$ is current pressure, $\mathrm{N} / \mathrm{m}^{2} ; p_{0}$ is maximal possible pressure, $\mathrm{N} / \mathrm{m}^{2}$.

Influence of mass density $\left(\mathrm{kg} / \mathrm{m}^{3}\right)$

$\gamma=\left(1-\frac{\rho}{\rho_{0}}\right)^{1 / 2}=\left(1-\frac{M^{2} G^{3}}{c^{6}} \rho\right)^{1 / 2}$, where $\rho_{0}=\frac{c^{6}}{G^{3}} \frac{1}{M^{2}}$,

where $\rho$ is current mass density, $\mathrm{kg} / \mathrm{m}^{3} ; \rho_{0}$ is maximal possible mass density, $\mathrm{kg} / \mathrm{m}^{3}$.

Influence of specific energy density $\left(\mathrm{J} / \mathrm{m}^{3}\right)$ for volume $v=$ const

$$
\gamma=\left(1-\frac{E_{v}}{E_{v, 0}}\right)^{1 / 2}=\left(1-\frac{M^{2} G^{3}}{c^{8}} E_{v}\right)^{1 / 2}, \text { where } E_{v, 0}=\frac{c^{8}}{G^{3}} \frac{1}{M^{2}},
$$

where $m^{3}$ is specific current energy density, $\mathrm{J} / m^{3} ; E_{v, 0}$ is maximal possible energy pressure, $\mathrm{J} / \mathrm{m}^{3}$.

Influence of temperature (using an additional relation $E=\frac{3}{2} k_{B} t$ ):

$\gamma=\left(1-\frac{t}{t_{0}}\right)^{1 / 2}=\left(1-\frac{k_{B}}{c^{2} M} t\right)^{1 / 2}$, where $t_{0}=\frac{c^{2}}{k_{B}} M$,

here $t$ is temperature, ${ }^{\circ} \mathrm{K} ; t_{0}$ is maximal possible temperature, ${ }^{\circ} \mathrm{K} ; k_{B}=1.38066 \cdot 10^{-23} \mathrm{~J} / \mathrm{K}$ is Boltzmann constant.

Influence of field frequency

$\gamma=\left(1-\frac{v}{v_{0}}\right)^{1 / 2}=\left(1-\frac{G M}{c^{3}} v\right)^{1 / 2}$, where $v_{0}=\frac{1}{T}=\frac{c^{3}}{G} \frac{1}{M}=\left(G \rho_{0}\right)^{1 / 2},(7)$

here $v$ is field frequency, $1 / \mathrm{s} ; v_{0}$ is maximal possible frequency, $1 / \mathrm{s} ; \mathrm{m}^{3}$ is maximal possible pressure, $\mathrm{kg} / \mathrm{m}^{3}$.

Wave De-Broil (using the additional relation $v_{B}=E / h$ ):

$$
\gamma=\left(1-\frac{v_{B, 0}}{v_{B}}\right)^{1 / 2}=\left(1-\frac{h}{2 c^{2} M} \frac{1}{v_{B}}\right)^{1 / 2} \text {, where } v_{B, 0}=\frac{2 c^{2}}{h} M,(8)
$$

where $v_{B}$ is wave frequency, $1 / \mathrm{s} ; v_{B, 0}$ is maximal possible wave frequency, $1 / \mathrm{s} ; h$ is Planck constant, $\mathrm{J} \cdot \mathrm{s}$.

Influence of the electric intensity [N/C]

$$
\gamma=\left(1-\left(\frac{E_{e}}{E_{e, 0}}\right)^{2}\right)^{1 / 2}=\left(1-\frac{\varepsilon_{0} G^{3} M^{2}}{2 c^{8}} E_{e}^{2}\right)^{1 / 2}, \text { where } E_{e, 0}^{2}=\frac{2 c^{8}}{\varepsilon_{0} G^{3}} \frac{1}{M^{2}}
$$

here $E_{e, 0}$ is electric intensity $[\mathrm{N} / \mathrm{C}]$ or volt/meter $[\mathrm{V} / \mathrm{m}] ; E_{e, 0}$ is maximal electric intensity $[\mathrm{N} / \mathrm{C}] ; \varepsilon_{o}$ is electric constant, $\mathrm{F} / \mathrm{m}$.

Influence of the magnetic intensity $[\mathrm{A} / \mathrm{m}]$

$$
\gamma=\left(1-\left(\frac{H}{H_{0}}\right)^{2}\right)^{1 / 2}=\left(1-\frac{\mu_{0} G^{3} M^{2}}{2 c^{8}} H^{2}\right)^{1 / 2} \text {, where } H_{0}^{2}=\frac{2 c^{8}}{\mu_{0} G^{3}} \frac{1}{M^{2}}
$$

where $H$ is magnetic intensity $[\mathrm{A} / \mathrm{m}] ; \mu_{o}$ is maximal magnetic intensity $[\mathrm{A} / \mathrm{m}] ; \mu_{o}$ is magnetic constant, $\mathrm{H} / \mathrm{m}$.

Influence of the acceleration field $\left[\mathrm{m} / \mathrm{s}^{2}\right]$

$$
\gamma=\left(1-\left(\frac{a}{a_{0}}\right)^{2}\right)^{1 / 2}=\left(1-\left(\frac{4 M G}{c^{4}}\right)^{2} a^{2}\right)^{1 / 2}, \text { where } a_{0}=\frac{c^{4}}{4 G} \frac{1}{M} \text {, }
$$

here $a$ is acceleration, $\mathrm{m} / \mathrm{s}^{2} ; r_{s}<i$ is maximal acceleration.

Influence of the distance from the center of the central point gravitation field, $r_{S}<r$ :

$$
\gamma=\left(1-\left(\frac{r_{S}}{r}\right)\right)^{1 / 2}=\left(1-\left(\frac{2 G M}{c^{2}}\right) \frac{1}{r}\right)^{1 / 2}, \text { where } r_{S}=\frac{2 G}{c^{2}} M
$$

where $r$ is distance from the center of the central gravitation field, $\mathrm{m} ; r_{S}$ is radius of Schwarzschild, $\mathrm{m}$.

Influence of the distance from center of the central electric field, $r_{E}<r$ (for charges only):

$$
\gamma=\left(1-\left(\frac{r_{E}}{r}\right)^{1 / 2}\right)^{1 / 2}=\left(1-\left(\frac{k_{e} Q^{2} G}{c^{4}}\right)^{1 / 2} \frac{1}{r}\right)^{1 / 2}, \text { where } r_{E}^{2}=\frac{k_{e} Q^{2} G}{c^{4}}
$$

here $r$ - distance from the center of the central electrostatic field, $\mathrm{m} ; r_{E}$ - event horizon of the central electrostatic field, $r_{E}<r, \mathrm{~m}$; $k_{e}=1 / 4 \pi \varepsilon_{0} \approx 9 \cdot 10^{9}$ - electric constant, $\mathrm{Nm}^{2} / \mathrm{C}^{2} ; Q$ - electric charge of body in given volume, $\mathrm{C}$.

Note: The maximal possible magnitudes are given an precision with numerical issue/multiplier (about $10^{ \pm 1}$ ). This value is found from testing/measuring or additional estimation. For example, the maximal possible mass density in expression (4) is

$$
\rho_{0}=\left(\frac{3}{32 \pi}\right) \frac{c^{6}}{G^{3}} \frac{1}{M^{2}} .
$$

Substitute the kinetic energy $E=M V^{2}(V$ is speed, $\mathrm{m} / \mathrm{s})$ in expression (2) we get the well-known expression of the Einstein special relativistic theory:

$$
\frac{T}{T_{0}}=\sqrt{1-\frac{V^{2}}{c^{2}}}, \quad \frac{l}{l_{0}}=\sqrt{1-\frac{V^{2}}{c^{2}}},
$$

here $V$ - speed of a moving body, $\mathrm{m} / \mathrm{s} ; l$ - time in a moving system, sec;,$l$ - length in a moving system, $\mathrm{m} ; T_{0}$ - time in stationary system, sec; $l_{0}$ - length in stationary system, $\mathrm{m}$;

Note, the expressions (2) - (13) are major differently from the relativistic equations (15). Expressions (15) measure the length and time of the body in a moving system of coordinates. The expressions (2- (13) show how changing the state of the motionless body that body can roll the size and time into point.

The numerical values of these limits in equations (2) - (13) are following (accuracy about 4 digits):

$$
\begin{aligned}
& p_{0}=\frac{c^{8}}{G^{3}} \frac{1}{M^{2}}=2.1962 \cdot 10^{98} \frac{1}{M^{2}}, N / \mathrm{m}^{2} ; \quad \rho_{0}=\left(\frac{3}{32 \pi}\right) \frac{c^{6}}{G^{3}} \frac{1}{M^{2}}=2.440 \cdot 10^{81} \frac{1}{M^{2}}, \frac{\mathrm{kg}}{\mathrm{m}^{3}} \\
& E_{v, 0}=\frac{c^{8}}{G^{3}} \frac{1}{M^{2}}=2.1962 \cdot 10^{98} \frac{1}{M^{2}}, \frac{\mathrm{J}}{\mathrm{m}^{3}} ; \quad \quad_{B, 0}=\frac{2 c^{2}}{\mathrm{~h}} M=2.71277 \cdot 10^{50} \mathrm{M}, \frac{1}{\mathrm{~s}} ; \\
& v_{0}=\frac{c^{3}}{G} \frac{1}{M} 4.002 \cdot 10^{35} \frac{1}{M}, \quad \frac{1}{\mathrm{~s}} ; \quad t_{0}=\frac{\mathrm{c}^{2}}{k_{B}} M=6.509607 \cdot 10^{39} \mathrm{~K} \\
& E_{e, 0}^{2}=\frac{2 c^{8}}{\varepsilon_{0} G^{3}} \frac{1}{M^{2}}=3.25144 \cdot 10^{110} \frac{1}{M^{2}}, \frac{\mathrm{N}}{\mathrm{C}}, \frac{\mathrm{V}}{\mathrm{m}}, \frac{\mathrm{kg} \cdot \mathrm{m}}{\mathrm{s}^{3} \mathrm{~A}}
\end{aligned}
$$




$$
\begin{aligned}
& H_{0}^{2}=\frac{2 c^{8}}{\mu_{0} G^{3}} \frac{1}{M^{2}}=3.40276 \cdot 10^{90} \frac{1}{M^{2}}, \quad T, \frac{W b}{m^{2}}, \frac{V s}{m^{2}}, \frac{N}{m A} ; \\
& a_{0}=\frac{c^{4}}{4 G} \frac{1}{M}=3.02639 \cdot 10^{43} \frac{1}{M}, \frac{m}{s^{2}} ; \quad r_{S}=\frac{2 G}{c^{2}} M=1.485676 \cdot 10^{-27} M, m ; \\
& r_{E}=k \frac{Q^{2} G}{c^{4}} \approx 7.4 \cdot 10^{-35} Q^{2}, m ; \quad E_{0}=c^{2} M=8.98752 \cdot 10^{16} M, J \\
& F_{0}=M a_{0}=3.02639 \cdot 10^{43} N .
\end{aligned}
$$

Here $F_{0}$ is maximal force, N. The temperature and maximal force are constants; they do not depend from mass.

As you see, the values are very minor or the values are very great. The real conditions are very far from collapse state. Rolling the space and time and in point (zero) may be in very minor volume (nuclear or less) or into a large mass of the huge density. The closed conditions may be in the wormholes, black holes, neutron stars and dwarfs.

Remain: the values (16) - (19) are calculated without the individual issue $\approx 10^{ \pm 1}$. This factor $( \pm 1)$ is minor in comparison to exponents $98,81,41$, etc., and may be found from the additional circumstances or experiment.

\section{Comment}

The calculated critical magnitudes are of great importance for micro and macro worlds. When a particle (body, universe) approaches them, for an external observer their dimensions, energy and lifetime tend to zero, i.e. they seem to disappear (they disappear into infinity). Because they depend on matter (energy), this means that matter (energy) and its parameters in the universe are limited.

\section{Discussion and conclusion}

In Part $1^{1}$ author shows: in the University is only ONE substance ENERGY. Only energy makes other known forms: time, space, matter, gravitation, magnetic, electric, nuclear fields. This result formed a new view of dark matter, dark energy, extension and acceleration of the Universe. ${ }^{1-8}$

Core result the Part 2 of this research is that each form or state of energy (density of matter, density of energy, frequency, temperature, density/intensity of the gravity, magnetic, electric, nuclear fields) have a LIMIT. When we are forthcoming this limit, the time, space (length, distance, volume) collapse (roll up) in point.

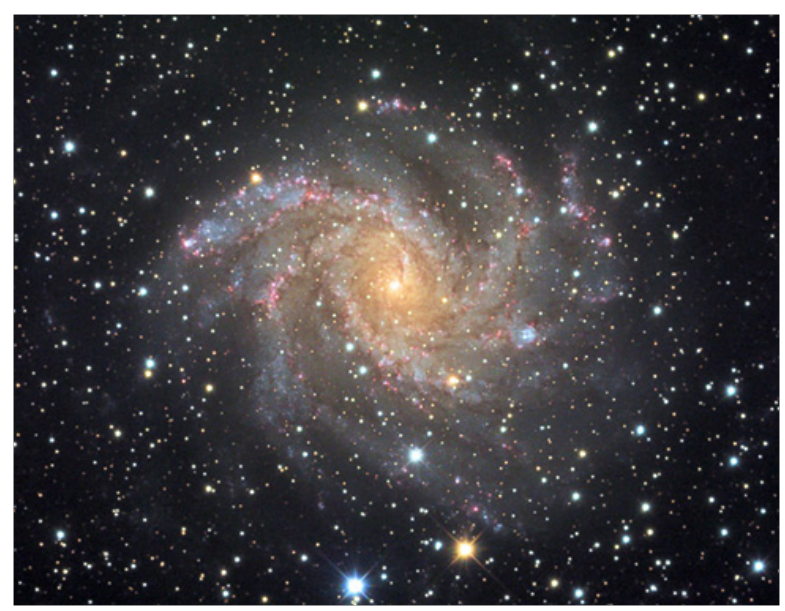

Figure I This image of the Hubble Ultra-Deep Field shows a diverse range of galaxies, each consisting of billions of stars.
The offered expression (2) - (13) are basically different from the relativistic equations (15). Equations (15) measure the length and time of a body in a moving system of coordinates. The equation (2) - (13) shows how we must change the conditions (pressure, density, temperature) of the motionless body or intensity/density of the magnetic, electric, gravitation/acceleration or centrifugal fields that body collapse (the time and size) in point for the outside observer. The critical state can continue indefinitely.

The critical value/limit may be high. But our abilities increase over time. We can study strong fields in the micro world. We can better recognize the macro/micro processes in the world.

The find limits are others or absent for negative mass, negative energy. In this case we may obtain repel (negative) gravity, the fasterthan-light speed, ${ }^{8}$ unlimited energy from vacuum point, exotic matter, and so on, which may help to develop the power spaceships for the interstellar travels, or to explain the inflation of the Universe. The computed critical values are of big importance for macro and micro worlds. When a particle (in universe or body) approaches them, for an external observer their energy, dimensions and lifetime tend to zero, i.e. they seem to disappear. They depend on matter (energy). This means that matter (energy) in the universe is limited (Figure 1).

\section{Acknowledgements}

None

\section{Conflict of interest}

Authors declare there is no conflict of interest.

\section{References}

1. Bolonkin AA. Universe. Relations between Time, Matter, Volume, Distance, and Energy (Part 1). Journal of Energy Storage and Conversion (JESC). 2012;3(2):141-154.

2. Bolonkin AA. Non Rocket Space Launch and Flight. Netherlands:Elsevier; 2006. p. 488.

3. Bolonkin AA. Preon Interaction Theory and Model of Universe. 2nd ed. Lulu; 2017. p. 105.

4. Bolonkin AA. New Concepts, Ideas, Innovations in Aerospace, Technology and the Human Sciences. NewYork: Nova Science Publishers; 2006. p. 510 .

5. Bolonkin AA. New Technologies and Revolutionary Projects. Lulu; 2010. p. 324.

6. Bolonkin AA. Converting of Any Matter to Nuclear Energy by-ABGenerator. American Journal of Engineering and Applied Science. 2009;2(4):683-693.

7. Bolonkin AA. Universe, Human Immortality and Future Human Evaluation. Lulu; 2010. p. 124.

8. Faster-than-light. 